\title{
Abbreviations and acronyms
}

Ancient Woodland Site

Any site that has a record of carrying tree cover since 1750 (in Scotland).

Ancient Semi-natural Woodland

Self-sown woodland of native tree species on an Ancient Woodland Site.

ATC - alternatives to clearfell

CCF - continuous-cover forestry

Methods of forest management that do not involve clearfelling/restocking.

Coppice management

Coppice-with-standards management

Cyclical woodland management systems relying on natural stump regrowth.

FC(S) - Forestry Commission (Scotland)

Government forest regulatory department/public forest management body.

NVC - National Vegetation Classification

A formal system for botanical description of semi-natural vegetation types.

PAWS - Plantations on Ancient Woodland Sites

Plantations of non-native tree species on Ancient Woodland Sites.

SNH - Scottish Natural Heritage

Government (statutory) nature conservation agency/nature reserve manager.

NTS - National Trust for Scotland

SWT - Scottish Wildlife Trust

WT - Woodland Trust

Non-governmental (charitable) bodies owning/managing woodland sites. 